\title{
ABOUT THE ACCELERATION RATE OF RELATIVISTIC BEAMS BY A SURFACE WAVE IN A DIELECTRIC LASER ACCELERATOR
}

\author{
O.O. Bolshov, A.V. Vasiliev, A.I. Povrozin, G.V. Sotnikov \\ National Science Center "Kharkov Institute of Physics and Technology", Kharkiv, Ukraine \\ E-mail: sotnikov@kipt.kharkov.ua
}

\begin{abstract}
An analysis of the dependence of the acceleration rate of charged particles by a surface wave arising when a laser pulse/(plane wave) is incident on the interface between two dielectric media on the phase velocity of the excited wave is carried out. It is shown that at resonance acceleration this dependence has a maximum, for ultra-relativistic particles the acceleration rate tends to zero. The dependences of the acceleration rate on the phase velocity of the excited wave for various refractive indices (dielectric permittivities) of optically transparent medias are investigated analytically and numerically.
\end{abstract}

PACS: 41.75.Lx, 41.60.-m, 41.75.Ht, 41.85.Ar

\section{INTRODUCTION}

The idea of dielectric laser accelerators (DLA), to use dielectric structures for acceleration by waves arising from their illumination with laser beams, arose in the last century [1-2]. Subsequent investigations showed the possibility of creating on the basis of this idea compact accelerators with aacceleration rate of $\sim 1 \mathrm{GeV} / \mathrm{m}[3-8]$. Interest in the use of laser beams to accelerate charged particles has renewed in the last decade due to the widespread use of lasers at the TW level of the micron wavelength and pulse durations 100fs and less. Progress in laser technology has made it possible to proceed to experimental verification and implementation of the basic principles of accelerators based on dielectric structures (see [9 - 12] and reference there).

The existing in NSC KIPT the TW laser system [13] also makes it possible to carry out research on laser acceleration in dielectric or chip structures. Due to the phase mismatch between the accelerated beam and the laser-excited wave, the creation of an operating accelerator will inevitably require its sectioning with different input beam energies. Our numerical simulation of the acceleration of beams in chip structures did not reveal a significant difference in the acceleration rates as a function of the initial beam energy [14, 15]. At the same time, it was stated in paper [2], and it was theoretically shown in paper [3] that for ultra-relativistic electrons the acceleration rate tends to zero, $E_{a c c} \propto \sqrt{1-\beta_{0}^{2}}=1 / \gamma_{0}$, $\beta_{0}$ is a phase velocity of incident wave. Similar dependence on relativistic factor $\gamma_{0}$ is also given in [16, 17] when studying DLA based on chip or grating structures.

The pointed out dependence strongly limits the possibilities of using the DLA scheme in the relativistic region of energies of the accelerated beams. In order to study the exact dependence of the acceleration rate on the beam energy and mitigate the limitations in the relativistic region, we, following the statement of problem [3], will obtain the amplitude of the transmitted wave when the plane wave is incident on the dielectricvacuum interface and will investigate it dependence on the beam velocity and dielectric material.

\section{STATEMENT OF THE PROBLEM AND BASE EQUATIONS}

Let an elliptically polarized plane wave falls from a optically transparent medium with refractive index $n_{1}=n$ under an angle $\theta$ onto the boundary between the vacuum and this medium. Geometry of the problem and the coordinate system is given in Fig. 1. For simplicity of perception in Fig. 1 are depicted only electromagnetic fields components corresponding to the case of $\mathrm{p}$ polarized plane wave. The boundary surface is the plane $y=0$, the incident plane is $x z$-plane, and the $\mathrm{x}$-axis is directed along the propagation of accelerated beam. In such a frame the components of the electric vector of the incident arbitrary elliptical wave will be of the form

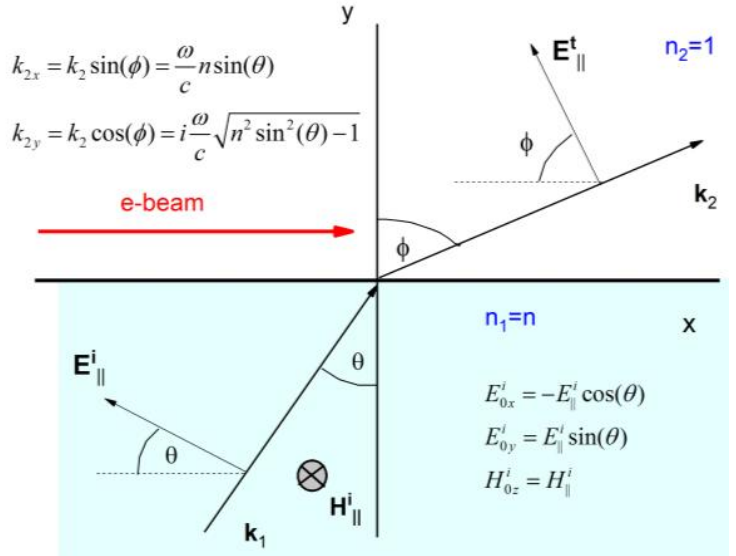

Fig. 1. Geometry of the problem and coordinate system. Here is shown the case of p-polarized plane wave is incident from dielectric medium $(y<0$, refractive index $\left.n_{1}=n\right)$ on the dielectric-vacuum interface $(y=0)$.

The accelerated electron beam moves parallel to the dielectric-vacuum boundary

$E_{x}^{i}=E_{0 x}^{i} \exp \left[i\left(\Phi+\Psi_{2}\right)\right]=-E_{\|} \cos \theta \exp \left[i\left(\Phi+\Psi_{2}\right)\right] ;$

$E_{y}^{i}=E_{0 y}^{i} \exp \left[i\left(\Phi+\Psi_{2}\right)\right]=E_{\|} \sin \theta \exp \left[i\left(\Phi+\Psi_{2}\right)\right] ;$

$E_{z}^{i}=E_{\perp} \exp \left[i\left(\Phi+\Psi_{1}\right)\right]$

where $\Phi=k_{1 x} x+k_{1 y} y-\omega t=k_{2 x} x+k_{12} y-\omega t, \quad E_{\|}$and $E_{\perp}$ are amplitudes of p-and s-polarized waves and $\Psi_{1}$ and $\Psi_{2}$ are their arbitrary phases. 
If $\sin \theta>1 / n$ the incident electromagnetic wave undergoes total internal reflection and $\sin \phi=n \sin \theta>1$. Then the analytical continuation of Snell's law to the complex plane provides for the wave vector of the transmitted wave

$$
k_{2 x}=\frac{2 \pi}{\lambda} n \sin \theta, k_{2 y}=i \frac{2 \pi}{\lambda} \sqrt{n^{2} \sin ^{2} \theta-1},
$$

$\lambda$ is the vacuum wavelength.

Having used the Fresnel formulae for the transmission coefficients separately for p-polarized, s-polarized wave obtain their expressions for the case total internal reflection

$$
\begin{gathered}
T_{\|}=\frac{2 n \cos (\theta)}{\sqrt{n^{2}-1} \sqrt{n^{2} \sin ^{2} \theta-\cos ^{2}(\theta)}} e^{-i \phi_{\|}}, \\
T_{\perp}=\frac{2 n \cos (\theta)}{\sqrt{n^{2}-1}} e^{-i \phi_{\perp}} .
\end{gathered}
$$

Here

$$
\operatorname{tg}\left(\phi_{\|}\right)=\frac{n \sqrt{n^{2} \sin ^{2} \theta-1}}{\cos (\theta)}, \operatorname{tg}\left(\phi_{\perp}\right)=\frac{\sqrt{n^{2} \sin ^{2} \theta-1}}{n \cos (\theta)} .
$$

Using equations (1), (3), (4) the components of the electric field can be written as

$$
\begin{aligned}
E_{x}^{t} & =-T_{\|} E_{\|} \cos (\phi) \exp \left[i\left(\Phi+\Psi_{2}-\varphi_{\|}\right)\right], \\
E_{y}^{t} & =T_{\|} E_{\|} \sin (\phi) \exp \left[i\left(\Phi+\Psi_{2}-\varphi_{\|}\right)\right], \\
E_{z}^{t} & =E_{\perp} T_{\perp} \exp \left[i\left(\Phi+\Psi_{1}-\varphi_{\perp}\right)\right]
\end{aligned}
$$

where $\Phi=\frac{\omega}{c} n \sin (\theta) x+i \frac{\omega}{c} \sqrt{n^{2} \sin ^{2} \theta-1}-\omega t$.

Separating the real part from expressions (5), we obtain the final expressions for the components of the electric field of transmitted wave

$$
\begin{aligned}
& E_{x}^{t}=E_{\|} F_{2} \sqrt{1-\beta_{0}^{2}} \sin \left(\Phi_{2}\right), \\
& E_{y}^{t}=E_{\|} F_{2} \cos \left(\Phi_{2}\right), E_{z}^{t}=E_{\|} F_{1} \cos \left(\Phi_{1}\right),
\end{aligned}
$$

and for components of the magnetic field

$$
\begin{aligned}
& H_{x}^{t}=-E_{\perp} \frac{F_{1}}{\beta_{0}} \sqrt{1-\beta_{0}^{2}} \sin \left(\Phi_{1}\right), \\
& H_{y}^{t}=-E_{\perp} \frac{F_{1}}{\beta_{0}} \cos \left(\Phi_{1}\right), H_{z}^{t}=E_{\|} F_{2} \beta_{0} \cos \left(\Phi_{2}\right) .
\end{aligned}
$$

In equations $(6),(7)$

$$
\begin{aligned}
F_{1}= & \frac{2 n \cos (\theta)}{\sqrt{n^{2}-1}} \exp \left[-\frac{2 \pi}{\lambda} \sqrt{n^{2} \sin ^{2}(\theta)-1} y\right], \\
F_{2}= & \frac{2 n^{2} \sin (\theta) \cos (\theta)}{\sqrt{n^{2}-1} \sqrt{n^{2} \sin ^{2}(\theta)-\cos ^{2}(\theta)}} \\
& \times \exp \left[-\frac{2 \pi}{\lambda} \sqrt{n^{2} \sin ^{2}(\theta)-1} y\right],
\end{aligned}
$$

$\Phi_{1}=\frac{2 \pi}{\lambda}[n \sin (\theta) x-c t]+\Psi_{1}-\varphi_{\perp}$,

$\Phi_{2}=\frac{2 \pi}{\lambda}[n \sin (\theta) x-c t]+\Psi_{2}-\varphi_{\|}, \quad \beta_{0}=\frac{1}{n \sin (\theta)}$.

The equations give the possibility to investigate the dependence of amplitude of transmitted wave on phase velocity surface wave $\beta_{0}$ arising when plane wave is incident to dielectric boundary interface undergoing the total internal reflection. It should be pointed that the expressions for the components of the electromagnetic fields of transmitted wave (6)-(8) coincide in form with given in [3]. More general case, when the angle between incident wave and direction of beam propagation is arbitrary, considered in [8]. However in the limiting case of zeroth this angle (this case correspond to the highest acceleration rate) the expressions [8] go to equations (6)-(8).

\section{NUMERICAL ANALYSIS}

As stated above, we will be interested in the dependence of the acceleration rate on the phase velocity of the synchronous wave, or on the initial beam energy. For beams propagating parallel to the dielectric-vacuum interface in the $\mathrm{x}$-axis direction, this acceleration rate $G_{\max }$ is determined by the amplitude of the longitudinal electric field component

$$
G_{\max }=q E_{\|} F_{2}\left(\beta_{0}\right) \sqrt{1-\beta_{0}^{2}}=q E_{\|} F_{a c} .
$$

In Fig. 2 are shown the dependencies of the amplitude of transverse component $F_{2}$ and longitudinal component $F_{a c}$ of transmitted wave from phase velocity of the excited wave at the boundary surface $y=0$ for the dielectric material of fused silica $n=1.453$.
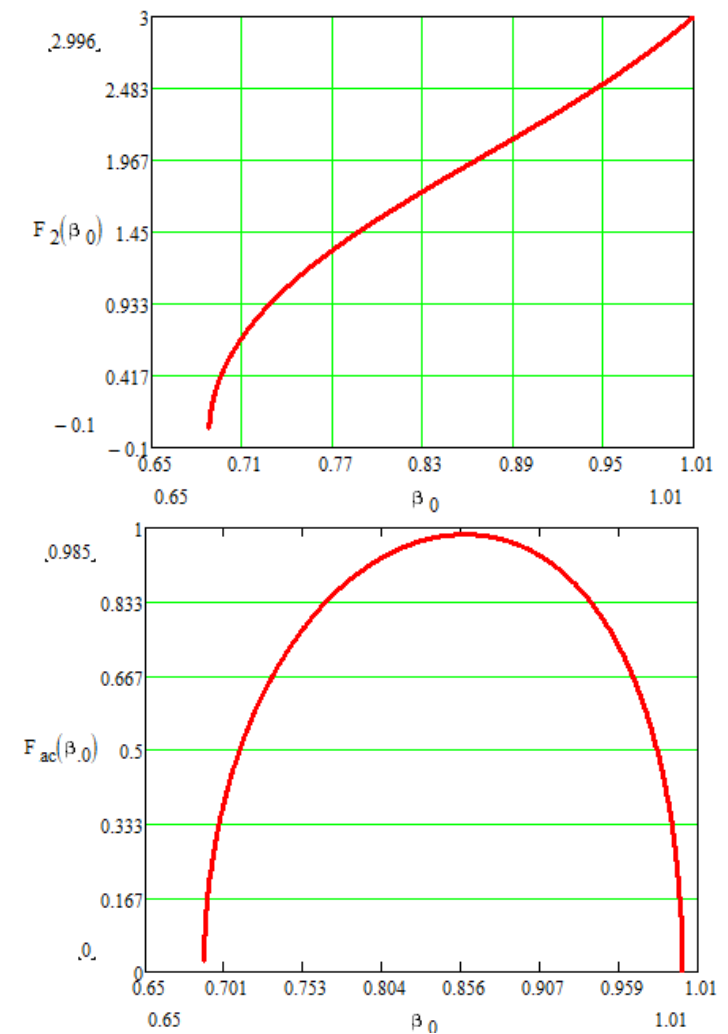

Fig. 2. Amplitude of transverse component $F_{2}$ (at the top) and longitudinal component $F_{a c}$ (at the bottom) of transmitted wave at the boundary surface versus phase velocity of the excited surface wave.

Permittivity of dielectric is $\varepsilon=2.112$ (fused silica)

From Fig. 2 follows that the transverse component amplitude of transmitted wave monotonically increases over entire interval of permitted $\beta_{0}$ when increase the phase velocity of excited surface wave. At the same time the longitudinal electric field increases from $\beta_{0}=1 / n \approx 0.7$ to $\beta_{0}=0.858$ (this corresponds electron beam energy $W_{b} \approx 0.5 \mathrm{MeV}$ ) and then falls quickly to 
zero ${ }^{1}$. When $W_{b} \approx 2.1 \mathrm{MeV}\left(\beta_{0}=0.858\right)$ acceleration rate decreases twice.

If we chose a different distance over the dielectric plane the then dependencies of transverse component $F_{2}$ and longitudinal component $F_{a c}$ remain qualitatively the same (Fig. 3 for $y=\lambda / 2$ ). The maximum of accelerated field is shifted to greater beam energies (compare with Fig. 2) and corresponds to beam energy $W_{b} \approx 1.6 \mathrm{MeV}$ $\left(\beta_{0}=0.97\right)$. At $W_{b} \approx 50 \mathrm{MeV}$ acceleration rate falls by 10 times in comparison with the maximum.
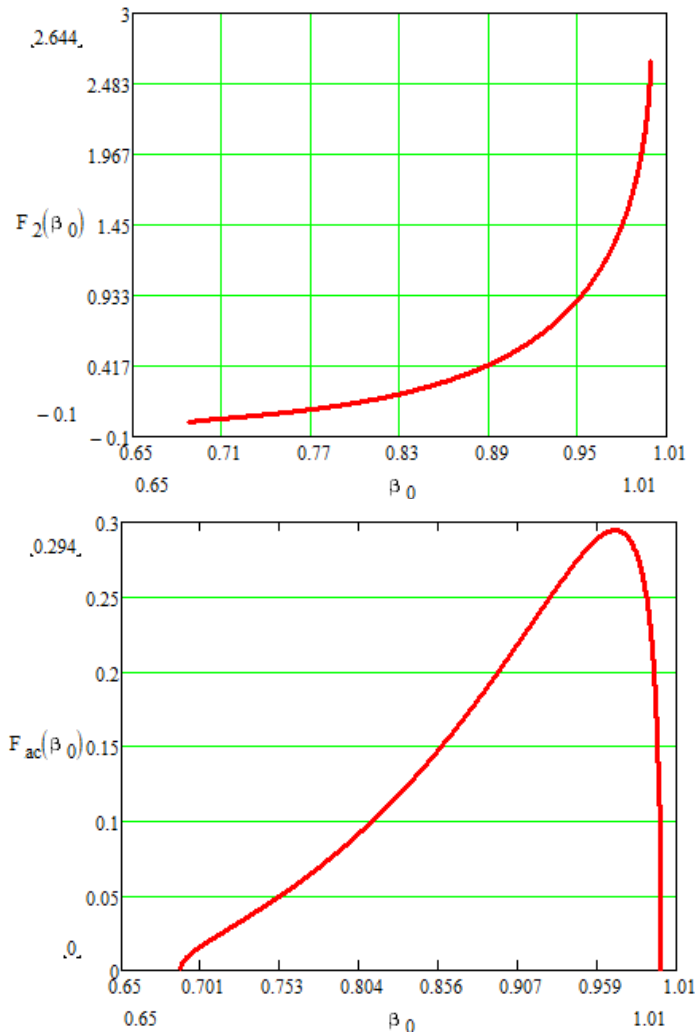

Fig. 3. Amplitude of transverse component $F_{2}$ (at the top) and longitudinal component $F_{a c}$ (at the bottom) of transmitted wave at $y=\lambda / 2$ versus phase velocity of the excited surface wave. Permittivity of dielectric is $\varepsilon=2.112$ (fused silica)

Now let us study the behavior of the longitudinal component of the electric field with a change in the dielectric medium. In Fig. 4 are shown the dependencies $F_{a c}$ on the phase velocity of excited surface wave for some optically transparent material: fused silica, sapphire, diamond, schott-IG, gallium indium arsenide and silicon-germanium. With an increase in the dielectric constant, the acceleration rate increases over the entire range of permitted phase velocities of the wave (the top graph), and its maximum shifts towards higher values of the phase velocity (the bottom graph). The latter result is especially important when accelerating more energetic electron beams. For case of silicon-germanium medium at $W_{b} \approx 50 \mathrm{MeV}$ acceleration rate falls by 8 times in comparison with the maximum.

Let us estimate the value of acceleration rate (9) in the best case from the presented in Fig. 4, the case of use for DLA of silicon-germanium medium.

${ }^{\mathbf{1}}$ While writing this paper we found that alike plotsare given in the paper [18].

ISSN 1562-6016. BAHT. 2021. №6(136)
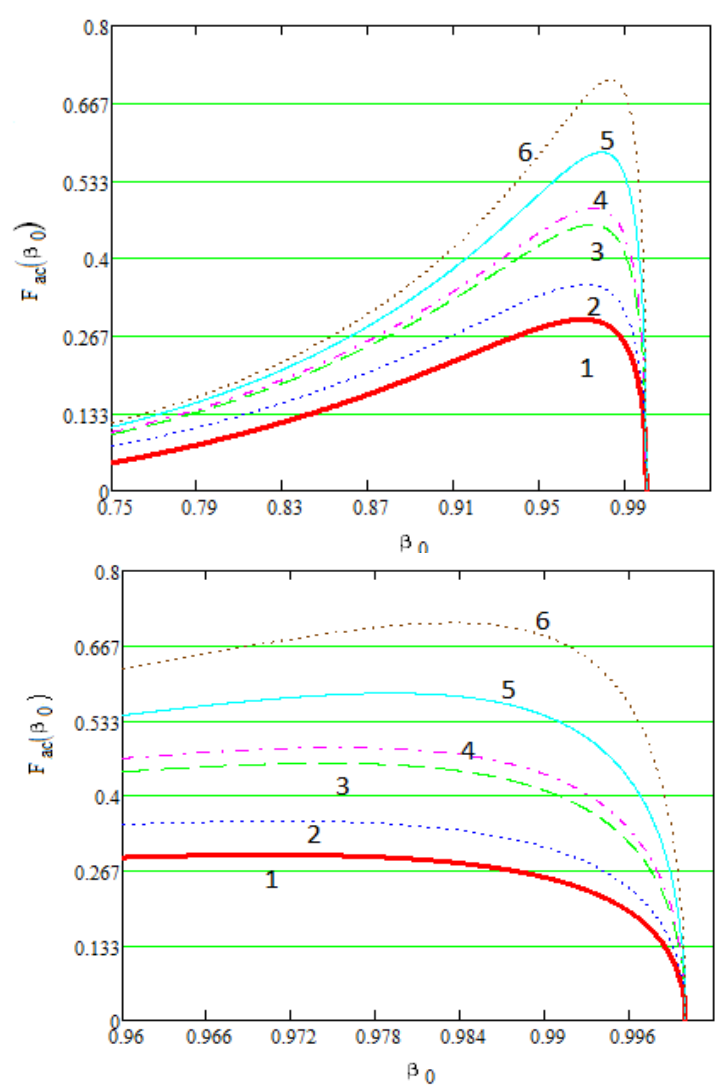

Fig. 4. Amplitude longitudinal component $F_{a c}$ (at the bottom) of transmitted wave at $y=\lambda / 2$ versus phase velocity of the excited surface wave: 1 -fused silica $\varepsilon=2.112$ - red line; 2 - sapphire $\varepsilon=3.1329$ - blue dotted line; 3 -diamond $\varepsilon=5.76$ - green dashed line; 4 - schott-IG $\varepsilon=6.7081$ - violet dot-dash line; 5 - gallium indium arsenide $\varepsilon=11.05496$ - cyan line; 6 - silicon-germanium $\varepsilon=20.196036$-brown dotted line

Let the amplitude of incident wave is $1.82 \mathrm{GeV} / \mathrm{m}$ (this corresponds the vacuum power density of $\left.4.4 \cdot 10^{11} \mathrm{~W} / \mathrm{cm}^{2}\right)$. In the maximum of acceleration rate (beam energy is $2.4 \mathrm{MeV}$ ) the acceleration rate is $1.3 \mathrm{GeV} / \mathrm{m}$. At $W_{b} \approx$ $50 \mathrm{MeV}$ acceleration rate is $160 \mathrm{MeV} / \mathrm{m}$.

\section{CONCLUSIONS}

I this paper the dependence of the acceleration rate of charged particles by a surface wave arising when a laser pulse/(plane wave) is incident on the interface between two dielectric media on the phase velocity of the excited wave is studied.

The dependences of the acceleration rate on the phase velocity of the excited wave for various refractive indices (dielectric permittivities) of optically transparent medias are investigated analytically and numerically.

At resonance acceleration these dependencies have the maximum, for ultra-relativistic particles the acceleration rate tends to zero. Maximum of acceleration rate increases when refractive index increases.

Maximum of acceleration rate shifts to higher initial energy of electrons when refractive index increases. This result is very important for DLA using for beam acceleraton the surface wave arising when laser beam is incident onto dielectric-vacuum boundary under the angle of total internal reflection. 
The applicability of the conclusion about a decrease in the acceleration rate when using ultra-relativistic electronic beams to accelerators using a chip or grating structures requires a separate study.

\section{ACKNOWLEDGEMENTS}

The study is supported by the National Research Foundation of Ukraine under the program "Leading and Young Scientists Research Support" (project \# 2020.02/0299), by NAS of Ukraine program "Perspective investigations on plasma physics, controlled thermonuclear fusion and plasma technologies", project P-1/63-2020.

\section{REFERENCES}

1. Koichi Shimoda. Proposal for an Electron Accelerator Using an Optical Maser // Applied Optics. 1962, v. 1, p. 33-35.

2. A. Lohmann. Electron Acceleration by Light Waves // IBM Technical Note TN5. 1962, p. 169-182.

3. S.A. Kheifetz. Particle motion a surface on which total internal reflection of electromagnetic wave takes plase // Proc. 8-th Int. conf. Higt Energy Accel., CERN, Geneva. 1971, p. 597-599.

4. H.A. Nagorsky, A.Ts. Amatuni, W.M. Harutiunian. Resonance acceleration of charged particles by a surface wave arising at total internal reflection // Proc. $12^{\text {th }}$ Int. Conf. on High Energy Accelerators, Fermilab. 1983, p. 488-490.

5. J.D. Lawson. Laser accelerators: where dowestand? // Proc. Workshop on the Generation of High Fields for Particle Acceleration to Very High Energies. 1986, p. 3-9.

6. I.V. Borovsky, S.V. Zhylkov, N.A. Khyzhnyak, V.G. Papkovich. To the theory of laser accelerationover dielectric comb // Problems of Atomic Science and Technology. Series "Technics of Physical Experiment”. 1987, № 3(34), p. 69-70.

7. I.V. Borovsky, S.V. Zhylkov, N.A. Khyzhnyak, V.G. Papkovich. About the acceleration of the REB over a dielectric comb // Problems of Atomic Science and Technology. Series "Technics of Physical Experiment”. 1987, № 4(35), p. 68-69.

8. R.C. Fernow. Acceleration using total internal reflection // BNL Report. 1991, № 52290, p. 1-17.
9. E.A. Peralta et al. Demonstration of electron acceleration in a laser-driven dielectric microstructure // Nature. 2013, v. 503, p. 91-94.

10. J. Breuer and P. Hommelhoff. Laser-based acceleration of nonrelativistic electrons at a dielectric structure // Phys. Rev. Lett. 2013, v. 111, p. 134803.

11. R.J. England et al. Dielectric laser accelerators // Rev. Mod. Phys. 2014, v. 86, p. 1337-1389.

12. Li Sun, Weihao Liu, Jie Zhou, et al. GV $\cdot \mathrm{m}^{-1}$ on-chip particle accelerator driven by few-cycle femtosecond laser pulse // New Journal of Physics. 2021, v. 23, p. 063031(10).

13. A.V. Vasiliev, A.N. Dovbnya, A.M. Yegorov, et al. Works in the NSC KIPT on the creation and application of the cpa laser system // Problems of Atomic Science and Technology. Series "Plasma Electronics and New Methods of Acceleration”. 2018, № 4, p. 289-292.

14. V. Vasyliev, O. Bolshov, K. Galaydych, A. Povrozin, G.V. Sotnikov. Influence of the profile of the dielectric structure on the electric fields excited by a laser in dielectric accelerators based on chip // Proc. of 12th Int. Particle Acc. Conf. IPAC2021. Campinas, Brazil. 2021, p. 2026-2029, https:// jacow.org/ipac2021/papers/tupab247.pdf

15. A.V. Vasyliev, A.O. Bolshov, K.V. Galaydych, et al. Acceleration of electron bunches using periodic dielectric structures with and without coating // Problems of Atomic Science and Technology. Series "Plasma Electronics and New Methods of Acceleration”. 2021, № 4, p. 60-64.

16. John Breuer. Dielectric laser acceleration of nonrelativistic electrons at a photonic structure // Dissertation an der Fakultat fur Physik der Ludwig Maximilians Universit at Munchen. 2013, p. 118. https:// edoc.ub.unimuenchen.de/16147/1/Breuer_John.pdf

17. N. Schönenberger and P. Hommelhoff. Dielectric Laser Acceleration // arXiv:2008.03958v1 [physics.acc-ph] 10 Aug 2020.

18. M. Kozák, P. Beck, H. Deng, J. Mc Neur, et al. Acceleration of sub-relativistic electrons with an evanescent optical wave at a planar interface // Opt. Express. 2017, v. 25, p. 19195-19204.

Article received 09.10.2021

\section{О ТЕМПЕ УСКОРЕНИЯ РЕЛЯТИВИСТСКИХ ПУЧКОВ ПОВЕРХНОСТНОЙ ВОЛНОЙ В ДИЭЛЕКТРИЧЕСКОМ ЛАЗЕРНОМ УСКОРИТЕЛЕ}

\section{А.О. Большов, А.В. Васильев, А.И. Поврозин, Г.В. Сотников}

Проведен анализ зависимости темпа ускорения заряженных частиц поверхностной волной, возникающей при падении лазерного импульса/(плоской волны) на границу раздела двух сред, от фазовой скорости возбуждаемой волны. Показано, что при резонансном ускорении эта зависимость имеет максимум, для ультрарелятивистских частиц темп ускорения стремится к нулю. Аналитически и численно исследованы зависимости темпа ускорения от фазовой скорости возбуждаемой волны для различных показателей преломления (диэлектрических проницаемостей) оптически прозрачных материалов.

\section{ПРО ТЕМП ПРИСКОРЕННЯ РЕЛЯТИВІСТСЬКИХ ПУЧКІВ ПОВЕРХНЕВОЮ ХВИЛЕЮ У ДІЕЛЕКТРИЧНОМУ ЛАЗЕРНОМУ ПРИСКОРЮВАЧІ}

\section{О.О. Больиов, А.В. Васильєв, А.І. Поврозін, Г.В. Сотніков}

Проведено аналіз залежності темпу прискорення заряджених частинок поверхневої хвилею, що виникає при падінні лазерного імпульсу/(плоскої хвилі) на межу розділу двох середовищ, від фазової швидкості хвилі, що збуджується. Показано, що при резонансному прискоренні ця залежність має максимум, для ультрарелятивістських частинок темп прискорення прагне до нуля. Аналітично та чисельно досліджено залежності темпу прискорення від фазової швидкості хвилі, що збуджується, для різних показників заломлення (діелектричної проникності) оптично прозорих матеріалів. 